tions were measured on a Rigaku AFC four-circle diffractometer using graphite monochromatised $M o K \alpha$ radiation $\left(2 \theta \leqslant 50^{\circ}\right)$

The structure was solved by the heavy atom method using 1,171 reflections with $2 \theta \leqslant 30^{\circ}$. The three-dimensional electron density map calculated on the basis of coordinates of the iodine atom revealed the rough structure of the complex. The least-squares refinement of the atomic parameters which were estimated on the electron density map was unsuccessful. The accurate positions and directions of the six glucose units and the $p$-IAN molecule were determined by the block-diagonal rigid-body least-squares technique ${ }^{3}$. The $\mathrm{O}_{6}$ atoms and water molecules were found on a difference Fourier synthesis. In successive refinements, one of the $\mathrm{O}_{8}$ atoms was found to be disordered. Using 3,519 reflections with $\left|F_{0}\right| \leqslant 3 \sigma(F)$, the final block-diagonal least-squares refinement with anisotropic temperature factors reduced the $R$ value to 0.072 . Fig 1 illustrates the structure of the complex.

Each glucose unit is in the $\mathrm{Cl}$ chair conformation, and all glucose units are $\alpha-1,4$-linked; this result is in agreement with the structures of the other $\alpha-C D x$ complexes which were determined by $\mathrm{X}$-ray analysis ${ }^{3-5}$. The $p$-IAN molecule is included in the cavity along the axis of $\alpha$-CDx. The iodine atom and the benzene ring are situated in the cavity of $\alpha-C D x$, while the nitrogen atom lies outside the cavity. The shortest distance between the benzene ring and $\alpha-C D x$ is $3.25 \AA$ which is from an $\mathrm{O}_{4}$ atom to a carbon atom of the benzene ring. This rather short intermolecular distance indicates that $p$-IAN is rigidly fixed in the cavity. The shortest distance from the iodine atom is $3.70 \AA$ which is the distance to the disordered $\mathrm{O}_{6}$ atom.

In the $\alpha$-CDx-p-IAN complex, the six $\mathrm{O}_{4}$ atoms of $\alpha$-CDx lie very near the least-squares plane of themselves, and the maximum deviation from the plane is $0.13 \AA$. In the $\alpha-C D x-$ potassium acetate complex ${ }^{3}$ and the $\alpha-C D x-H_{2} \mathrm{O}$ complex ${ }^{4}$, the maximum deviations are $0.01 \AA$ and $0.98 \AA$, respectively. The average valence angle of $\alpha-1,4$-linking oxygen atoms is $120^{\circ}(2)$ which is in agreement with $119.1^{\circ}$ in the $\alpha$-CDx-potassium acetate complex and $119 \pm 3^{\circ}$ in the $\alpha-\mathrm{CDx}-\mathrm{I}_{2}$ complex ${ }^{5}$. The distances between oxygen atoms $\mathrm{O}_{2}$ and $\mathrm{O}_{3}$ of adjacent glucose units are $2.68,2.76,3.00,2.92,2.98$ and $2.87 \AA$ which are the acceptable distances for hydrogen bonds. On the other hand, in the $\alpha-\mathrm{CDx}-\mathrm{H}_{2} \mathrm{O}$ complex and the $\alpha-\mathrm{CDx}-\mathrm{I}_{2}$ complex, there exist distances of $3.36 \AA$ and $3.83 \AA$, respectively. In the $\alpha$-CDx-p-IAN complex, all $\mathrm{C}_{6}-\mathrm{O}_{6}$ bonds are oriented to the outside of the cavity and the conformation is the same as that found in the $\alpha-C D x-I_{2}$ complex; the conformational angles $\mathrm{O}_{5}-\mathrm{C}_{5}-\mathrm{C}_{8}-\mathrm{O}_{6}$ are $-75 \pm 8^{\circ}$ in the $\alpha$-CDx-p-IAN complex and $-70 \pm 8^{\circ}$ in the $\alpha-\mathrm{CDx}-\mathrm{I}_{2}$ complex.

Research Institute for Polymers and Textiles,

KazUaki HaRata Hisashi UedaIRA

4, Sawatari, Kanagawa-ku, Yokohama,

Japan

Received September 2, 1974

1 French, D., Levine, M. L., Pazur, J. H., and Norberg, E., J. Am. chem. Soc., 71, 353 (1949).

2 Demarco, P. V., and Thakker, A. L., Chem. Commun., 2 (1970).

3 Hybl, A. Rundle, R. E., and Williams, D. E., J. Am. chem. Soc., 87, 2779 (1965).

4 Manor, P. C., and Seanger, W., Nature, 237, 393 (1972).

5 McMullan, R. K., Seanger, W., Fayos, J., and Mootz, D., Carbohyd. Res., 31, 211 (1973).

\section{Is the aardwolf a mimic of the hyaena?}

MIMICRY is a phenomenon of evolutionary convergence or parallelism by which an edible mimic species gains some measure of protection from predators by virtue of its close resemblance to a model species which is unpalatable (that is, distasteful or dangerous $)^{1-3}$. Predators learn to avoid the unpalatable model species after one or more adverse experiences with it, and any mimic species that resembles the model sufficiently closely will likewise be avoided.

The first examples of mimicry were documented in Brazilian butterflies ${ }^{1}$ - most additional examples and experimental work have been limited to mimicry in insects. Vertebrates exhibiting mimicry are rare, but include fish $^{4}$, snakes ${ }^{5,6}$ and birds ${ }^{7,8}$. A single example has previously been described in mammals: Shelford described a series of five squirrel species which mimic unpalatable treeshrew models in Borneo ${ }^{8,10}$. The case described here is a possible additional example which, if confirmed, extends the phenomenon of mimicry to a large African mammal.

The genus Hyaena is represented in Africa today by two species: the striped hyaena, $H$. hyaena, inhabiting northern Africa; and the brown hyaena, $H$. brunnea, limited to southern Africa. Hyaenas (the spotted hyaena, Crocuta, excluded) weigh $50-60 \mathrm{~kg}$, and inhabit open dry plains and thorn scrub, live singly or in pairs, and are chiefly nocturnal. Hyaena has a sloping back, pointed ears, and an erectile mane. It has strong
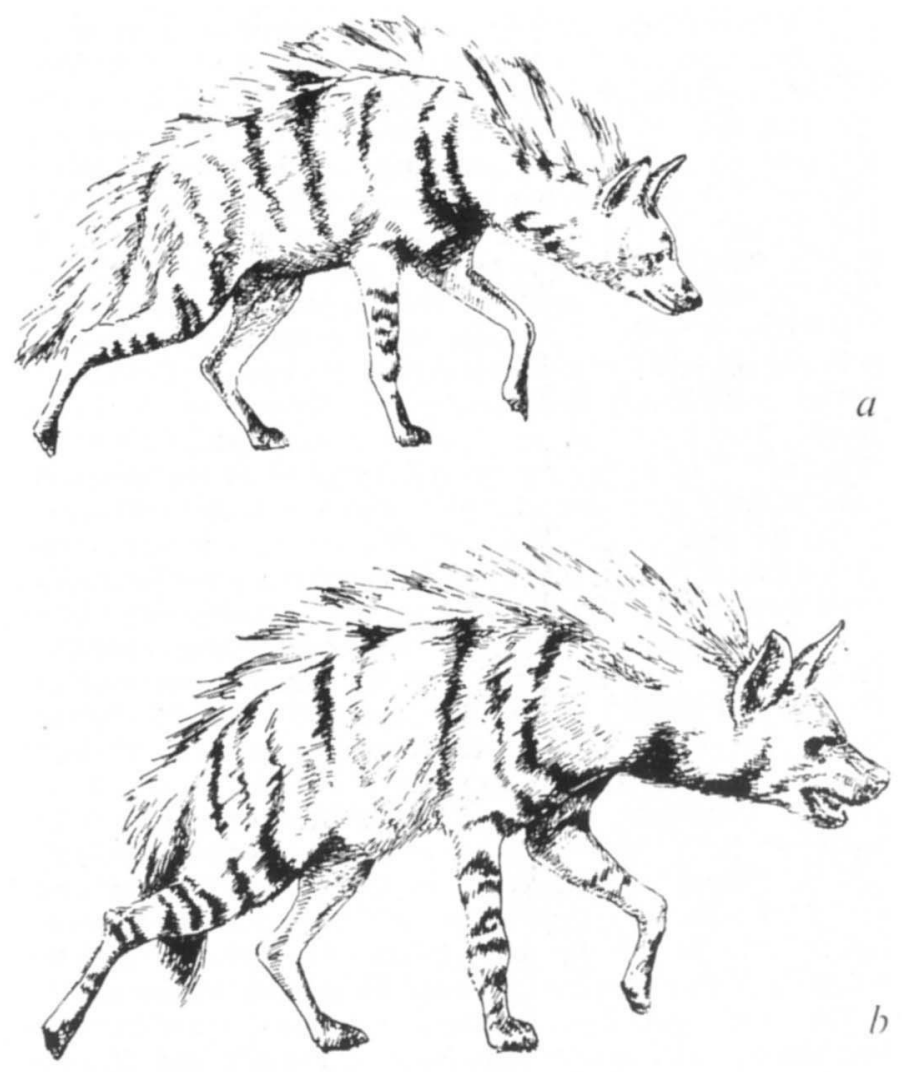

Fig. 1 External appearance of, $a$, the aardwolf, Proteles cristatus, and, $b$, the hyaena, Hyaena hyaena, both drawn to the same scale. Redrawn by $\mathbf{P}$. Olsen from colour illustrations in Dorst and Dandelot ${ }^{29}$

teeth and jaws and it is known to kill live game occasionally, although it is primarily a scavenger.

The aardwolf Proteles cristatus is a much smaller, jackelsized $(12-15 \mathrm{~kg})$ mammal with a discontinuous distribution: a northern population is found throughout most of East Africa, and a separate population inhabits southern Africa. Proteles, like Hyaena, has a sloping back, pointed ears, and a well developed erectile mane along the dorsal spine from neck to tail. This mane is composed of stiff hairs some $20 \mathrm{~cm}$ in length and, when erected, makes the aardwolf appear considerably larger than it actually is ${ }^{11}$. Proteles inhabits open 
plains and thorn scrub, lives singly or in pairs, and is chiefly nocturnal. It has only vestigial cheek teeth and feeds largely on termites and other insects, and occasionally on carrion and rodents ${ }^{12,13}$.

In his initial description of Proteles, Geoffroy St-Hilaire ${ }^{14}$ noted that its external appearance is very similar to that of Hyaena, a resemblance noted in virtually every subsequent description of the aardwolf (Fig. 1). Kruuk ${ }^{13}$ observed that Proteles and Hyaena look so similar that they are often confused. The resemblance of Proteles to Hyaena in body, mane and tail colour, and in the colour and development of stripes remains close in spite of considerable clinal variation in these characters; the pattern in each subspecies of Proteles resembling closely the sympatric subspecies of Hyaena ${ }^{14-16}$. Size is the only real difference in the external morphology of the two, and this is a notoriously difficult quantity to perceive in the field in the absence of some fixed comparative scale.

In addition, Proteles is found throughout its distribution in the same open plain or thorn scrub habitat as Hyaena, and its general behaviour is remarkably similar. The aardwolf and hyaena are both chiefly nocturnal, and live singly or in pairs. Even their kneeling defensive response to attack is similar ${ }^{11}$.

The external similarity of Proteles to Hyaena extends also to most of the characters of internal anatomy that have been studied, indicating that Proteles is closely related to the hyaenas. The chromosomes ${ }^{17-19}$ and haemoglobin mobility ${ }^{20}$ of Proteles are virtually identical to those of Hyaena and Crocuta, and the gyri and sulci of the cerebral hemispheres of the brain are arranged on exactly the same plan ${ }^{11}$. The complete dental formula of Proteles, though not always fully developed, is the same as that of Hyaena and Crocuta ${ }^{21}$. The male reproductive tract of Proteles appears to be more similar to that of Hyaena than to that of Crocuta ${ }^{11,22}$. Proteles differs from Hyaena principally in having a dentition much reduced in size, and in retaining the pollex (a digit lost in both Hyaena and Crocuta) ${ }^{23}$.

The evolutionary lines leading to Hyaena and Crocuta appear, from the fossil record, to have been distinct since the Miocene ${ }^{24}$. Fossils bearing on the evolution of the aardwolf have recently been discovered ${ }^{21,25}$ which indicate a Pliocene or earlier time of divergence of Proteles from the ancestral hyaenid stock, but the precise phylogenetic relationships among these three genera of Hyaenidae are not yet completely clear.

Two hypotheses of hyaenid relationships seem tenable. Proteles may have separated from the common ancestor of Crocuta and Hyaena before they separated (suggested by the shared loss of the pollex in the latter two genera), in which case the close external resemblance of Proteles to Hyaena is perhaps convergent. On the other hand, Crocuta may have separated from the Proteles-Hyaena stock before these two became differentiated (suggested by the reproductive tract and the close external resemblance), in which case the external resemblance of Proteles and Hyaena may reflect a parallel retention from a common ancestor having this appearance.

The nearly complete geographic sympatry, close external resemblance, and similar behaviour of Proteles and Hyaena are unusual in closely related forms, since speciation normally involves significant divergence in geographic distribution, in external appearance, or in behaviour. All of the conditions of Batesian mimicry are met by Proteles, and this may help to explain its distribution, appearance, and behaviour. Only the predator remains to be identified.

Leopards occur throughout the range of Proteles, they are most active at night, and they are predominantly visually oriented predators. Leopards routinely prey on jackels ${ }^{26,27}$ and, in view of their wide prey tolerance ${ }^{28}$, might be expected to prey on Proteles as well. Because of its large size and strong skull, a Hyaena would be dangerous to an attacking leopard. The much smaller, weaker Proteles would be a poor match for a leopard, and it seems that an important component of the aardwolf's defence against predators may be its close external resemblance to the larger, more dangerous Hyaena. The ability of Proteles to erect its mane when excited would further confuse a potential predator as to its actual size and identity.

This possible case of mimicry in a large mammal is unique, and deserves further investigation. Unfortunately, low population densities and nocturnal activity patterns make both Proteles and Hyaena difficult to study in the field. Additional study of their behaviour and ecology will be of great interest, and observation of any interactions with leopards of particular importance. Museum of Paleontology,
The University of Michigan,
Ann Arbor, Michigan 48104

Philip D. Gingerich



THE intensity of body and tissue protein metabolism per $\mathrm{kg}$ declines with increased adult body size in mammals ${ }^{1}$. This fall parallels a similar progressive decline in the intensity of energy metabolism ${ }^{2-4}$. It has also been concluded that protein metabolism per unit of body weight is about four to five times faster in young rats than in adult man ${ }^{1}$; this pattern of change extends to cellular and subcellular aspects of protein metabolism, such as plasma albumin synthesis, liver RNA content and enzyme activity $^{1,5}$. Similarly, the rate of protein synthesis per $\mathrm{kg}$ total body weight declines during growth and development within a species, such as the rat ${ }^{6}$. This parameter again parallels the reduction in the intensity of energy metabolism which occurs during the growth period ${ }^{3}$.

Such surveys provide a general picture of protein metabolism in mammals and help to explain the metabolic basis for differences in dietary protein requirements both within and between the various species. The concepts involved should apply to man at various ages ${ }^{7-9}$. In previous studies with human subjects, however, different approaches have been used, some of which are no longer considered valid, and insufficient detail has been provided. It is therefore difficult to use the estimates to compare changes in body protein and energy metabolism with those for dietary protein needs throughout a man's life.

Using a common approach, we have tried to characterise the relationship which exists between protein and energy metabolism in man and to examine dynamic aspects of body protein metabolism with reference to dietary protein needs, during the period of rapid growth and development and the later years when senescence dominates body metabolism and function. The procedures, analytical methods and calculations of total body 